\title{
CULTURA DEL PATRIMONIO PRIVADO EN EL ESTADO DE TABASCO
}

\author{
CULTURE OF PRIVATE HERITAGE IN THE STATE OF TABASCO
}

\author{
Rubí Cadena-Valenzuela ${ }^{1 *}$. \\ 1. Escuela Judicial del Estado de Tabasco, México. rubi-cadena-valenzuela@hotmail.com \\ * Autor de correspondencia: Rubí Cadena Valenzuela, correo electrónico: rubi-cadena-valenzuela@hotmail.com
}

\section{RESUMEN:}

La escasa cultura del patrimonio privado, es una de las circunstancias que originan conflicto entre particulares que terminan en largos litigios en los Tribunales. Aunado a las escasas políticas públicas para su protección, ha sido el campo propicio para que pseudo gestores inmobiliarios, abusen de las personas que de buena fe ponen en sus manos el patrimonio que con toda una vida de esfuerzo han construido.

La cultura del patrimonio privado es importante para la calidad de vida de las personas y para una empresa es fundamental para sobrevivir y continuar aportando a la activación económica y generación de empleos.

Es por ello que, al momento de realizar cualquier transacción sobre el patrimonio privado de las personas, cuando menos deben de estar informados de cuáles son las disposiciones básicas para llevar a cabo su acto jurídico y que tengan certeza de la propiedad que adquieren, y evitar problemas a futuro.

Palabras clave: Cultura; patrimonio privado; accesión; adjudicación; sucesión; usucapión; prescripción positiva; ocupación; el contrato; la ley.

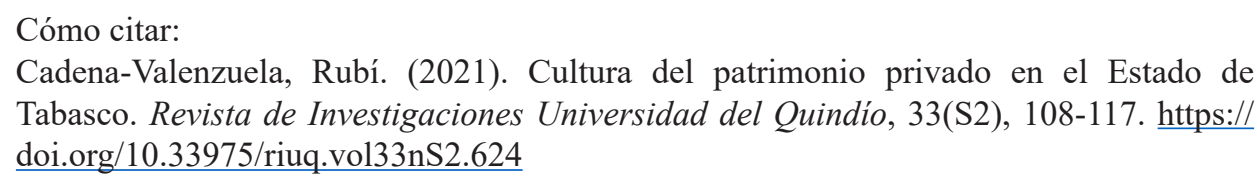




\begin{abstract}
The scarce culture of private heritage is one of the circumstances that originate conflict between individuals that end in long litigation in the Courts.

In addition to the scarce public policies for their protection, it has been the propitious field for pseudo real estate managers to abuse people who in good faith put in their hands the heritage that with a lifetime of effort they have built.

The culture of private heritage is important for the quality of life of people and for a company it is essential to survive and continue contributing to the economic activation and generation of jobs.

That is why, at the time of carrying out any transaction on people's private assets, at least they must be informed of what the basic provisions are to carry out their legal act and that they have certainty of the property they acquire, and avoid future problems.
\end{abstract}

Keywords: Culture; private equity; accession; award; succession; usucapion; positive prescription; occupation; contract; the law.

\title{
INTRODUCCIÓN
}

Este trabajo de investigación pretende acrecentar la cultura en cuanto al patrimonio privado para que a través de campañas de concientización se advierta a las personas de posibles defraudadores que abusan del desconocimiento de los procesos de adquisición o transacciones inmobiliarias que casi siempre van en detrimento de las clases más desfavorecidas social y culturalmente.

Si bien es cierto existe una amplia información en medios electrónicos, también es de reconocer que para la gente común dichas operaciones inmobiliarias les resultan tediosas y costosas lo que origina una opacidad para concluir sus procesos de adquisición.

Además, que aún cuando los notarios son funcionarios del estado que velan por los intereses patrimoniales de las personas ayudando a prevenir fraudes, se acostumbra en la práctica que las personas creen que el documento que les otorga el vendedor al realizar la transacción es suficiente para transmitir la propiedad, cuando en realidad no produce efectos jurídicos frente a terceros, puesto que faltan otros actos jurídicos como pueden ser su protocolización e inscripción ante el Registro Público de la Propiedad.

Siendo así una puerta abierta para que duplique o triplique la venta apareciendo varios dueños quienes creen que su documento o título que poseen los acredita como propietarios, al existir varios poseedores la Ley toma como base la que primera que esté inscrita en el Registro Público de la Propiedad, y es allí donde los demás propietarios se creen desposeídos de sus patrimonios que con tanto esfuerzo invirtieron durante toda su vida, lo que ocasiona una injusticia social.

Por todo ese desconocimiento para la adquisición y protección de la propiedad privada, los Tribunales Civiles del Estado de Tabasco, se ven saturados por tanto conflictos sobre posesiones y propiedades, mismos que de la práctica diaria arroja un porcentaje del treinta al cuarenta por ciento de los juicios ventilados en los Juzgados Civiles, versan sobre propiedades privadas. 


\section{FUNDAMENTO CONSTITUCIONAL DE LA PROPIEDAD PRIVADA}

La adquisición de una propiedad es una de las inversiones más importante que podemos hacer en nuestras vidas.

Es un derecho consagrado en al artículo 27 de la Constitución General de la República, en donde se habla de tres tipos de propiedad, la propiedad pública, la propiedad privada y la propiedad social.

La propiedad pública se refiere al derecho que tiene el Estado Mexicano (Federación, Estados, Municipios), sobre bienes de dominio público que son aquellos bienes muebles e inmuebles que están sujetos a un régimen de derecho público, están fuera del comercio, son inalienables, imprescriptibles e inembargables. Los bienes del dominio público pueden ser bienes de uso común, bienes destinados a un servicio público y bienes propios.

Ahora bien, la propiedad social se compone de diversos grupos agrarios y a su vez por dos regímenes: la propiedad ejidal y la propiedad comunal que son distintas a la propiedad privada.

Y tenemos también la propiedad privada que es a la que nos vamos a referir en este artículo y es el derecho que tiene una persona física o moral particular para gozar y disponer de sus bienes con las limitaciones establecidas por la ley.

De tal forma que una persona física o moral particular podrá ser propietario tanto de bienes muebles como bienes inmuebles dentro con las limitaciones establecidas por la ley.

Nuestro Código Civil del Estado de Tabasco, conceptualiza a la propiedad en el artículo 951, teniéndolo como un derecho real que otorga a una persona, el poder jurídico para usar, gozar o disponer de una cosa, pero dentro de las limitaciones y con arreglo a las leyes.

Las formas de adquirir la propiedad, pueden ser por contrato, herencia, usucapión o prescripción positiva, adjudicación, accesión y la Ley.

Todas estas terminologías pueden resultar confusas para la mayoría de la población que no está familiarizados con ello, por lo mismo es importante simplificar y dejar claro las diferencias entre estas formas de transmitir la propiedad.

El contrato es una transmisión a título particular y casi siempre onerosa en la que el adquiriente paga cierto valor, bienes o servicios, a cambio de un bien que recibe.

La herencia es una transmisión por causa de muerte y puede revestir dos formas: testamentaria o intestamentaria.

Usucapión y/o prescripción positiva es acto de declaración unilateral de la voluntad en el que la autoridad judicial declara la adquisición de un_derecho real_, es decir de la propiedad, mediante la posesión que exija la ley, y ¿Qué exige la Ley? que sea originaria y probar la existencia del título que la genere, debe ser pacífica, continua y publica, para los bienes inmuebles el término debe ser de cinco años poseídos de buena fe. 
Adjudicación es una transmisión a título particular donde se le atribuye una cosa o un bien, a una persona a través de una subasta, licitación o partición hereditaria.

La adquisición por medio de la Ley, puede ser la donación o herencia.

Para que un contrato sea capaz de producir la traslación de dominio, es necesario otro acto jurídico configurado para tal efecto, por ello el artículo 1295 del Código Civil del estado en su primer párrafo estable los títulos sujetos al Registro público serán: “...I. Los títulos por los cuales se adquiera, transmitan, modifique, grave o extinga el dominio, la posesión o los demás derechos reales sobre inmuebles..."

El contrato de compraventa requiere para su validez, formalidad especial cuando recae sobre un inmueble.

La venta de un inmueble que tenga un valor equivalente hasta la cantidad de quinientos días de salario mínimo general vigente en el Estado, podrá hacerse en documento privado que firmarán el vendedor y el comprador ante dos testigos, ratificado ante el director del Registro Público de la Propiedad, jefe de la oficina registral correspondiente o ante Notario Público. La compraventa de inmuebles, así como las hipotecas, que celebren las instituciones que tiene como objeto proporcionar viviendas terminadas o lotes de interés social, y que hayan sido creadas para cumplir esa finalidad, tanto por el Gobierno Federal como por el Estatal o Municipal, con beneficiados que sean de escasos recursos, podrá hacerse constar en documento privado en los términos del párrafo anterior, cualquiera que sea su costo. Para certeza de los contratos citados en el párrafo precedente, los funcionarios y directivos de tales instituciones, registrarán sellos y firmas en el Registro Público de la Propiedad y del Comercio del Estado.

\section{DELITOS PATRIMONIALES}

En la vida cotidiana las personas se ven inmersas en situaciones relacionadas con bienes de su propiedad o bienes que son propiedad de otros y surgen numerosas cuestiones en relación al derecho de propiedad, el alcance del mismo y sus efectos.

Cuando estas posesiones se realizan de mala fe, comúnmente terminan en las agencias ministeriales como delitos patrimoniales tales como despojo, fraude, extorsión y hasta robo, en el que después de múltiples y desgastantes diligencias por la vía penal el querellante solo obtiene, si bien le va, la reparación del daño, sin que realmente quede resuelto de fondo el conflicto.

E1 "paracaidismo" se ha vuelto una forma de adquirir viviendas en nuestro país, ya que existen familias o grupos grandes de personas que aprovechan inmuebles o terrenos desocupados por un largo periodo de tiempo, para apropiarse de ellos y establecer en dichos bienes ajenos sus casas o negocios constituyendo de esta manera el delito de despojo.

El delito de despojo se presenta principalmente cuando una persona o grupo de personas, privan a otra de la posesión de sus bienes inmuebles como lo son casas y terrenos, haciendo uso de violencia o amenazas en la mayoría de los casos.

La ley establece una pena o sanción de tres meses a cinco años de prisión y multa de cincuenta a quinientos pesos. 
Se comete un fraude de ley cuando se realiza un acto jurídico amparándose en una ley de cobertura con la intención de alcanzar objetivos impropios de esa norma y, además, contrarios a otra ley o al ordenamiento jurídico. Es decir, se utiliza una ley de cobertura para ocultar la ley defraudada. Además, el acto fraudulento debe buscar un fin condenado por otra norma del ordenamiento.

Los actos realizados al amparo del texto de una norma que persigan un resultado prohibido por el ordenamiento jurídico, o contrario a él, se considerarán ejecutados en fraude de ley y no impedirán la debida aplicación de la norma que se hubiere tratado de eludir.

En definitiva, se trata de camuflar o disfrazar una conducta como si fuera otra para burlar una norma. El Código Civil sanciona esta técnica de aplicación de una norma jurídica, una maniobra que la jurisprudencia suele calificar como "pseudo legal".

Esto se debe a que con esta técnica se intenta demostrar una legalidad aparente y, por el contrario, no se pretende cometer una infracción directa, lo que implicaría la nulidad radical del acto o negocio jurídico realizado. Algunos ejemplos clásicos del fraude de ley son las contrataciones de falsos autónomos o las compraventas que se disfrazan como donaciones con intención de defraudar la ley fiscal.

La extorsión es la presión que se ejerce sobre alguien mediante amenazas para obligarlo a actuar de determinada manera y obtener así dinero u otro beneficio.

Sinónimo de este concepto podría ser lo que encierra el delito de amenaza condicional. Sin embargo, según la jurisprudencia no sería correcto dar el mismo uso de extorsión a los términos de coacción o amenaza.

Dado que la extorsión es un delito pluriofensivo, no solo es un bien jurídico el que puede verse afectado, sino muchos más.

Así podemos afirmar que lo que el Código Penal protege tipificando la extorsión es: el patrimonio, la integridad física y la libertad.

Extorsionar consiste en obligar a alguien con violencia e intimidación a hacer o no hacer un acto o negocio jurídico en perjuicio de su patrimonio o del de un tercero.

El robo es un delito que consiste en apropiarse de los bienes o derechos de alguien por medio de la fuerza o por intimidación.

Se pueden distinguir dos tipos de robo: Robo con fuerza en las cosas, este tipo de robo se produce cuando existe escalamiento, rompimiento de pared, techo, suelo, puerta, ventana, armarios, muebles etc., utilización de llaves falsas, o bien inutilización de sistemas de alarma o guarda.

El robo con violencia o intimidación en las personas, Se produce cuando se utiliza la fuerza física, la violencia o las coacciones sobre las personas víctimas del delito para realizar el robo. 


\section{ELEMENTOS A CONSIDERAR PARA REALIZAR UNA COMPRAVENTA DE BIEN INMUEBLE}

Comprar un inmueble ya sea una casa, un terreno, un local comercial, requiere de informarse adecuadamente, pues existen muchos pasos y acciones que tomar en cuenta, además de que se involucran diferentes personas e instituciones.

Lo ideal es contar con el capital suficiente para comprar de contado, pero la mayoría de las veces se tiene que recurrir a créditos hipotecarios por medio de Instituciones privadas como las bancarias o por medio de Instituciones públicas como Instituto del Fondo Nacional para Vivienda de los Trabajadores (INFONAVIT) o el (FOVISSSTE), por lo que se debe considerar las diferentes etapas del proceso.

\section{Averiguar todo sobre el inmueble a comprar}

Si la casa es de segunda mano, lo primero es solicitar el título de la propiedad y asegurarse que el inmueble esté libre de adeudos o esté comprometida en otro crédito. Para ello, basta con acudir al Registro Público de la Propiedad para solicitar un documento que indique la situación de la casa ante esa institución.

También es útil consultar los portales inmobiliarios en México, que son sitios en internet que se enfocan en la venta, renta de bienes raíces, por citar algunos como CRM inmobiliario, Lamudi, nocnok, Inmuebles24, metroscubicos.com, entre otros; Son plataformas muy prácticas en la búsqueda de propiedades, que contiene fotografías y amplios catálogos para poder ver a detalle las características, ubicaciones, rangos de precios de los inmuebles.

\section{Comprobar si se cuenta con el financiamiento necesario}

Es necesario conocer el valor del inmueble. Evaluar las diferencias entre un crédito hipotecario de tasa fija y una de tasa creciente. El costo de un crédito es más que solo los intereses, pues es necesario calcular otros gastos fuera del valor de la casa. Más que la tasa de interés, evalúa el Costo Anual Total (CAT), un indicador porcentual anual que suma algunos costos extras.

\section{Calcular el enganche y otros gastos iniciales}

Entre más ahorro se destine para el enganche, menor será el financiamiento y los pagos mensuales. El enganche no es el único pago inicial, también se deben sumar otros gastos como el avalúo y los gastos notariales, entre otros, como seguro de vida que pagará la deuda en caso de fallecimiento, seguro de daños, que protegerá la casa de distintos daños que puedan ocurrirle.

Nunca se debe dar dinero a modo de enganche o depósito sin un contrato que formalice la operación.

\section{Los gastos de escrituración}

El notario redactará una escritura en la que se hará constar quien es el nuevo dueño del inmueble y debe verificar, entre otras cosas, que la persona que vende sea el dueño y que la propiedad esté regularizada. 
Una escritura pública es un documento público que se realiza ante un notario público, un determinado hecho o derecho autorizado, en donde firman el otorgante u otorgantes.

¿Qué es la Escrituración? Es hacer constar con un documento oficial un contrato del dominio total o parcial de un terreno o una propiedad, aunque este no se utilice, los beneficios que puede llegar a tener esto son muchos, tanto como ganancia propia vivienda, además te sirve para poder ampararse de alguna situación en caso de tener un imprevisto.

Actualmente el costo de los gastos de escrituras de compraventa de una vivienda oscila entre el 5\% y el $9 \%$ del valor total de propiedad.

La mayor proporción del costo son los impuestos y derechos que el notario retiene y tiene que pagar al fisco, si se quiere escriturar algo primero que nada debe haber un convenio entre comprador y la persona que vende para que se pueda llevarse a cabo el proceso, si esto llegara a ser una herencia familiar o de algún otro tipo el proceso es el mismo lo único que cambia son los trámites a realizar, sin embargo escriturar tiene un costo que muchas veces es caro.

Escriturar una propiedad trae varios beneficios y es por eso la importancia que tiene el ser dueño de una propiedad.

Para que se pueda asegurar legalmente una propiedad, el dueño debe realizar una escrituración, pues es la única forma de acreditar de manera legal la posesión de dicho inmueble, ya otro tipo de contrato como lo son los contratos privados no es reconocido ante la ley mexicana como válido.

\section{REQUISITOS PARA ESCRITURAR:}

Los documentos esenciales que piden cualquier notario público para escriturar una casa o un terreno son prácticamente los mismos:

- Escritura de antecedentes, de donde emana la transacción.

- Plano expedido por persona certificada con cedula profesional.

- Avalúo comercial, pagos de impuesto predial y valor catastral.

- Datos de la propiedad, dirección, superficie, medidas, linderos, colindancias

- Datos personales del vendedor. (INE, CURP, RFC, comprobante de domicilio)

- Datos personales de comprador. (INE, CURP, RFC, comprobante de domicilio)

\section{DEL REGISTRO PÚBLICO DE LA PROPIEDAD}

La institución. El Registro Público de la Propiedad, es la institución mediante la cual el Estado proporcionará el servicio de dar publicidad a los actos jurídicos que, conforme a las leyes, precisen de ese requisito para surtir efectos frente a terceros. El Ejecutivo del Estado designará las poblaciones en donde debe establecerse la oficina denominada "Registro Público de la Propiedad".

El Registro será público. Los encargados de la oficina tienen la obligación de permitir a las personas que lo soliciten, que se enteren de las inscripciones constantes en los Libros del Registro Público de la Propiedad y de los documentos relacionados con las inscripciones, que estén archivados. También tienen obligación de expedir copias certificadas de las inscripciones o constancias que figuren en los 
libros del Registro Público de la Propiedad, así como certificaciones de no existir asientos de ninguna especie o determinada, sobre bienes señalados o a cargo de ciertas personas.

Tratándose de testamentos ológrafos depositados en el Registro Público de la Propiedad, se observará lo dispuesto en el Capítulo correspondiente.

¿Qué se inscribirá? Se inscribirán en el Registro Público de la Propiedad: I. Los títulos por los cuales se adquiera, transmitan, modifique, grave o extinga el dominio, la posesión o los demás derechos reales sobre inmueble; II. La constitución del patrimonio de familia ...” entre otros.

\section{RESULTADOS Y ANÁLISIS}

El derecho civil es la rama del derecho privado constituida por un conjunto de normas que se refieren a las relaciones jurídicas de la vida del ser humano, familia y posesiones.

En materia civil y familiar existen muchas clases de juicios o procesos a través de los cuales se dirimen las controversias jurídicas, a eso se le conoce como vías procesales.

En la legislación del estado de Tabasco, existen cuatro vías, la vía ordinaria, la especial, vía Ejecutiva y Civil Oral, así como otro tipo de procedimientos como los no contenciosos.

Vía Ordinaria: por regla general todos los juicios que no tengan una tramitación específica, se tramitarán por esta vía. Se caracteriza por tener los plazos más largos. Ejemplos de juicios ordinarios: Juicio Ordinario Civil de Nulidad y cancelación de escritura, Juicio Ordinario Civil Otorgamiento y firma de escritura pública, Juicio Ordinario Civil de inexistencia de contrato de compraventa, Juicio Ordinario Civil de Cumplimiento de Contrato de Compraventa, Juicio Ordinario Civil de Rescisión de Contrato, Juicio Ordinario Civil Reivindicatorio, Juicio Ordinario de Usucapión, Acciones plenarias de Posesión, entre muchos más.

Vía sumaria o Especial: La Ley establece reglas específicas para su tramitación, por su naturaleza deben dirimirse de manera más pronta, tiene por objeto el cumplimiento de las obligaciones. Ejemplos de Juicios Especiales: Juicio Especial Hipotecario, Juicios Especial de desahucio, Juicio Especial de Cancelación de Hipoteca, Juicio Especial de Interdicción para recuperar la posesión, Juicio Familiar Especial de pensión alimenticia, Juicio Especial de Cancelación de Pensión Alimenticia, Juicios Sucesorios Testamentarios e Intestamentarios.

Vía Ejecutiva: versa sobre cualquier cuestión relativa sobre cumplimiento o ejecución, siempre que se trate de 1. Acción de condena que tenga por objeto exigir el pago de una suma de dinero cierta y determinada. II. Que la acción se funde en un título que traiga aparejada ejecución, y III. Que el adeudo sea cierto líquido y exigible.

Vía Oral: Su característica principal es que se desahogan las audiencias de manera oral y se acortan los tiempos de trámites.

Existen otros procedimientos Judiciales donde por solicitud de los interesados se requiere de la intervención del juzgador, sin que se promueva cuestión litigiosa alguna entre las partes, tales como: En materia civil se ventilan los medios preparatorios a Juicio, preliminares de la consignación, petición 
de medida cautelar, Informaciones Ad perpetuam Rei Memorian y/o Información de dominio para acreditar un derecho, Apeo y Deslinde, por citar algunos.

En materia familiar se ventilan los procedimientos de Adopción, Divorcios Voluntarios, Procedimiento de Autorización para gravar bienes y transigir derechos de menores, para sacar a menores de País, Declaración de Ausencia y Presunción de Muerte, entre otros.

A continuación, se presenta una gráfica que indica el porcentaje de los distintos Juicios que se ventilan en el estado de Tabasco, donde se podrá constatar que existe un porcentaje muy alto de los procedimientos que versan sobre propiedades, aparte de los del orden familiar que también es la gran mayoría.

Con ello se reafirma la preocupación que existan campañas de promoción de una cultura del patrimonio privado para evitar que seudos gestores inmobiliarios, o pseudo líderes abusen del desconocimiento de las personas para sacar ventajas de la necesidad comprar o vender sus bienes, ya que no existe una unidad pública o de interés social para acompañar a las personas o empresas en sus procesos de adquisiciones.

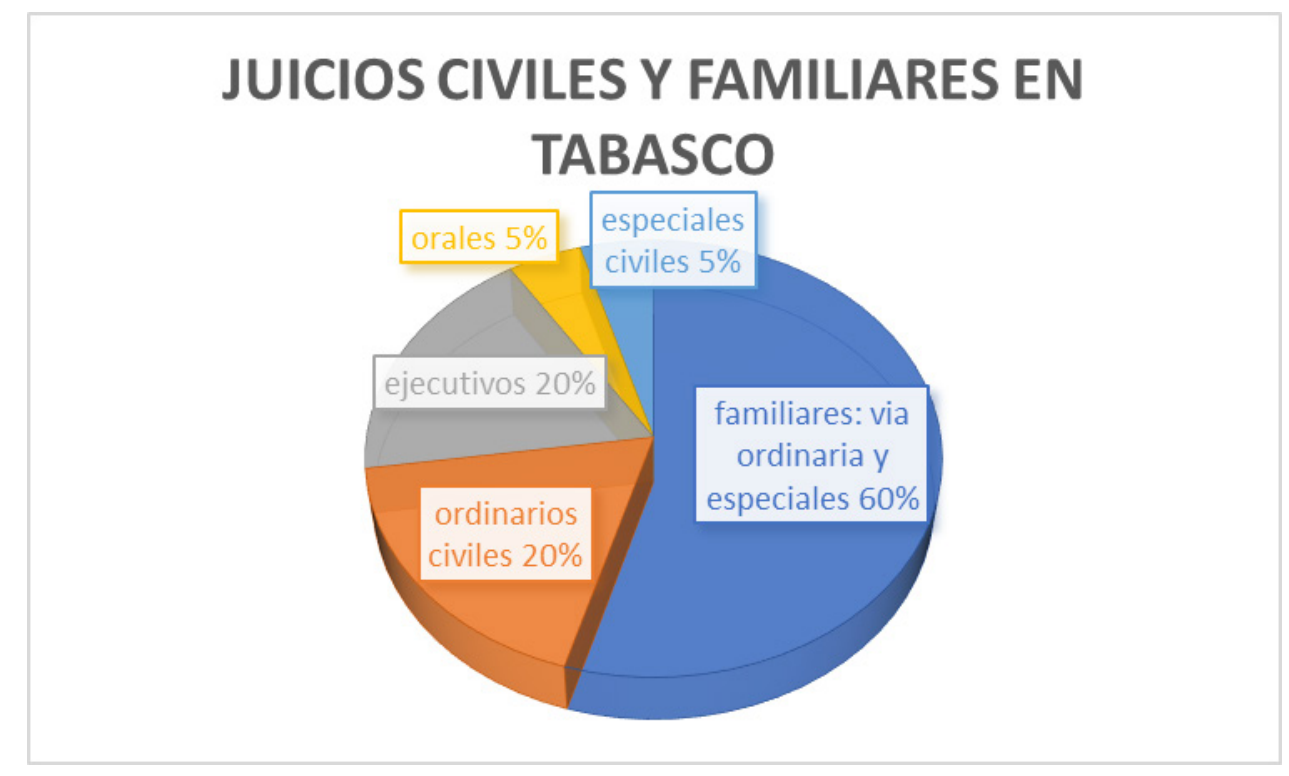

\section{CONCLUSIONES Y TRABAJO FUTURO:}

El objetivo de este artículo no es descubrir el hilo negro ni mucho menos, simple y sencillamente, es hacer consciencia que en el estado de tabasco, como en muchos otros estado de la República Mexicana, que están creciendo a pasos agigantados, debe ya existir un crecimiento poblacional ordenado, por lo que urgen realizar campañas de cultura del patrimonio privado, donde los ciudadanos tenga la certeza jurídica de las transacciones de sus bienes raíces que realiza.

Pareciera que en plena era tecnológica y de tanta información no fuera posible que existan personas que se dejan enganchar o extorsionar por gente que se dice experta en cuestiones inmobiliarias, le dejan su patrimonio y posesiones en gente a ventajosa, y es allí donde se comenten las injusticias sociales, pues se ven desposeídos de su patrimonio de toda una vida de lucha y esfuerzo.

Es por ello que el estado debe de fomentar esa cultura del patrimonio privado, haciendo promoción 
tal vez a través de portales oficiales, haciendo uso de las redes sociales, donde no se necesite invertir tanto recurso financiero, dado la accesibilidad de las redes sociales y televisoras públicas.

Con acciones simples se pueden evitar grandes problemas sociales y jurídicos, como lo son los largos y tediosos conflictos que se suscitan entre las empresas o personas por sus bienes.

Al tener un crecimiento ordenado y jurídicamente estable, tanto las empresas y las personas enfocan sus energías en ser realmente productivos, lo que trae consigo que la economía de la población se incremente y a la vez la Paz Social, que es el fin último de la Justicia.

\section{REFERENCIA}

1. Código Civil y de Procedimientos Civiles del estado de Tabasco. www.unam.mx 